\title{
Effectiveness of Redundant Communications Systems in Maintaining Operational Control of Small Unmanned Aircraft
}

\author{
Jaewoo Jung \\ Aviation Systems Division \\ NASA Ames Research Center \\ Moffett Field, USA \\ jaewoo.jung@nasa.gov
}

\author{
Sreeja Nag \\ Bay Area Environmental Research Institute \\ NASA Ames Research Center \\ Moffett Field, USA \\ sreeja.nag@nasa.gov
}

\author{
Hemil C. Modi \\ Science and Technology Corp. \\ NASA Ames Research Center \\ Moffett Field, USA \\ hemil.c.modi-1@nasa.gov
}

\begin{abstract}
As a part of NASA's Unmanned Aircraft System (UAS) Traffic Management (UTM) research, a test was performed to evaluate the effectiveness of the redundant Command and Control (C2) communications system for maintaining operational control of small UAS in the airspace over a rural area. In the test, operators set up a primary and a secondary UAS C2 communications system, sent a maneuver command to an Unmanned Aircraft (UA) with and without a functioning primary system, then verified the execution of the sent command to confirm the operator control. Operators reported that the tested redundancy configurations were effective in maintaining operational control in the test airspace over rural locations. Since the next phase of UTM research focuses on operations in an urban area where an increased level of Radio Frequency (RF) activities occur compared to a rural area, four recommendations are provided to sustain the effectiveness of redundancy in urban operations. First, the operator should not include $\mathrm{C} 2$ systems that use the industrial, scientific, and medical (ISM) radio bands in redundancy configurations. Second, the operator should verify the RF characteristics of the intended operation area and examine the area's radio noise floor. Third, the operator should monitor the availability, quality, and reliability of communications services used by a redundant system. Fourth, the small UAS community should adopt a standard set of contingency steps to handle the loss of $\mathrm{C} 2$ communications so that such events are managed in a consistent manner across the airspace. The insights from the test will be used to accommodate the FAA's UAS integration effort.
\end{abstract}

Keywords-operations, control, communications, redundancy

\section{INTRODUCTION}

NASA has been researching prototype technologies for an Unmanned Aircraft System (UAS) Traffic Management (UTM) system to facilitate safe and efficient civilian low-altitude airspace and UAS operations [1,2]. The UTM research accommodates the FAA's UAS integration effort, and the UTM system is designed to provide services at low altitudes where the FAA's Air Traffic Management (ATM) services are not available, therefore complementing the ATM system [3]. The FAA expects that the UTM capabilities will be incrementally implemented to address this gap over the next several years[4].
To effectively transfer NASA's UTM findings to the FAA, NASA and the FAA formed a Research Transition Team [5]. The team is divided into four subgroups, Concepts and Use Cases; Data Exchange and Information Architecture; Sense and Avoid; and Communications and Navigation (C\&N). Of the four subgroups, this paper discusses activities within the C\&N subgroup whose objective is to explore communications and navigation solutions to ensure that Unmanned Aircraft (UA) are under the operational control of the remote pilot and remain within a defined area. In particular, the paper describes a test that is designed to evaluate the effectiveness of redundant Command and Control (C2) communications systems in maintaining operational control of small UAS (sUAS) in the airspace over a rural area and presents a summary of the test reports. Since the next phase of UTM research focuses on sUAS operations in the airspace over an urban area, where a higher level of radio activities occur over broad Radio Frequency (RF) spectrum compared to a rural area [6], the paper also provides recommendations about configuring effective redundant $\mathrm{C} 2$ communications systems for urban operations.

\section{TEST DESCRIPTION}

In 2018, NASA contracted six FAA-designated UAS test sites to evaluate the UTM capabilities developed to date. In this evaluation, four of the six sites, Alaska, Nevada, New York, and North Dakota, performed a test to evaluate the effectiveness of the redundant $\mathrm{C} 2$ communications system, described in this paper, in maintaining operational control of sUAS in the airspace over several rural locations.

For the test, NASA provided the instruction shown in Table 1 to each Test Site Operator (TSO). The instructions were designed to show that sUAS equipped with redundant $\mathrm{C} 2$ communication systems could execute a maneuver command via either C2 system, maintaining operational control when one system is compromised. Given the variety of technologies and services that can be used for sUAS communications, the test did not specify a specific redundancy system and the TSOs used redundancy configurations of their own choosing. Table 2 shows a summary of the redundancy configurations used in the 
test. Note some similarities and differences between the redundancy setups. For example, most redundancy configurations involved two communication systems. The range of communication systems included radio, LTE cellular, Wi-Fi, and satellite. Most redundant systems identified a primary communication system with automatic switching to a secondary system when the primary fails. One automatically switched to the system providing the best data quality. One was switched manually by the operator. One merged the data from both systems.

The TSOs were required to conduct the test using at least three different flight plans that were significantly different in terms of takeoff location, takeoff time, altitude profile, and lateral flight profile. After the test, each TSO debriefed and reported on the test processes and results.

\section{SUMMARY OF TEST REPORTS}

\section{A. Alaska}

The Alaska flight test was conducted on April 25, 2018. Two quadcopters were flown in a box pattern in Fairbanks, at the locations shown in Figure 1 [7]. During the flights of a quadcopter with two identical radio modems, the $\mathrm{C} 2$ communications link established by one radio was manually disconnected by the operator to force a switchover to the other radio. During the flights of another quadcopter with an LTE cellular modem and a radio modem, the LTE C2 communications link failed without intervention from the operator and caused a switchover to the radio. The Alaska TSO reported that in both cases having a secondary $\mathrm{C} 2$ communications system improved reliability in maintaining the operator control.

\section{Table 1. Test Instruction}

\begin{tabular}{|c|c|}
\hline STEP & INSTRUCTION \\
\hline 1 & $\begin{array}{l}\text { Equip sUAS with more than one } \mathrm{C} 2 \\
\text { communications system }\end{array}$ \\
\hline 2 & Prepare UA to fly within a defined area \\
\hline 3 & Once UA is airborne, send a maneuver command \\
\hline 4 & Confirm execution of the sent command \\
\hline 5 & $\begin{array}{l}\text { Take one } \mathrm{C} 2 \text { system off-line (e.g., disconnect, } \\
\text { turn off signal, etc.) }\end{array}$ \\
\hline 6 & $\begin{array}{l}\text { Use redundancy to send another maneuver } \\
\text { command }\end{array}$ \\
\hline 7 & Confirm execution of the second sent command \\
\hline
\end{tabular}

\section{B. Nevada}

The Nevada flight test was conducted on March 9, 2018. A fixed-wing UA equipped with two different radio modems was flown in three planned patterns in North Valleys. Figure 2 shows the planned flight locations [8]. During a flight, the operator turned off the $\mathrm{C} 2$ communications signal of one radio to force a transition to the other radio. The Nevada TSO reported that the operator was able to maintain control with this transition throughout the test.

C. New York

The New York flight test was conducted on April 18-19, 2018. An octocopter UA equipped with a Wi-Fi modem and an

Table 2. Redundant sUAS C2 Communications System Configurations Used in the Test

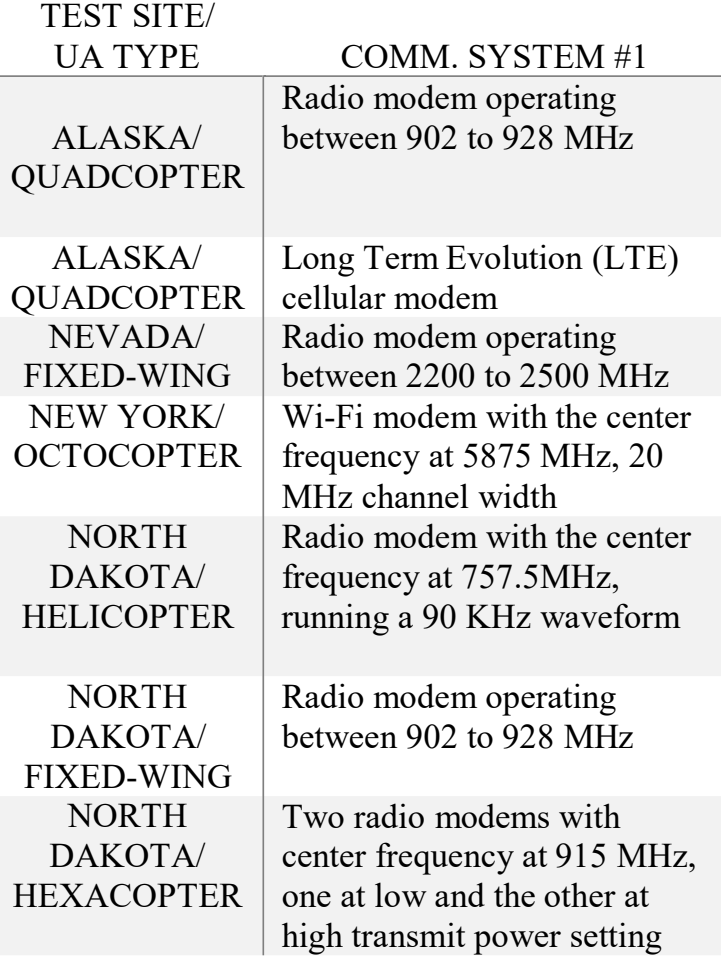

COMM. SYSTEM \#2

Radio modem operating between 902 to $928 \mathrm{MHz}$

Radio modem operating between 902 to $928 \mathrm{MHz}$ Radio modem operating between 2405 to $1470 \mathrm{MHz}$ LTE cellular modem

Radio modem with the center frequency at $907.5 \mathrm{MHz}$, running a $90 \mathrm{KHz}$ waveform

Satellite communication modem operating in 1616 to $1626.5 \mathrm{MHz}$ Dual LTE mobile modemrouter that connects to two different cellular services

\section{NOTE}

Each radio connected to a separate Ground Control System (GCS), \#2 link echoing \#1 link with automatic switching to \#2 when \#1 fails \#1 used as primary, automatic switching to \#2 when \#1 fails Operator switches between the two systems \#1 used as primary, automatic switching to \#2 when \#1 fails

Both radios transmit all data, automatically merging to a single receiving stream with duplicate data filtered out.

Automatic switching to the communications link with the best quality 


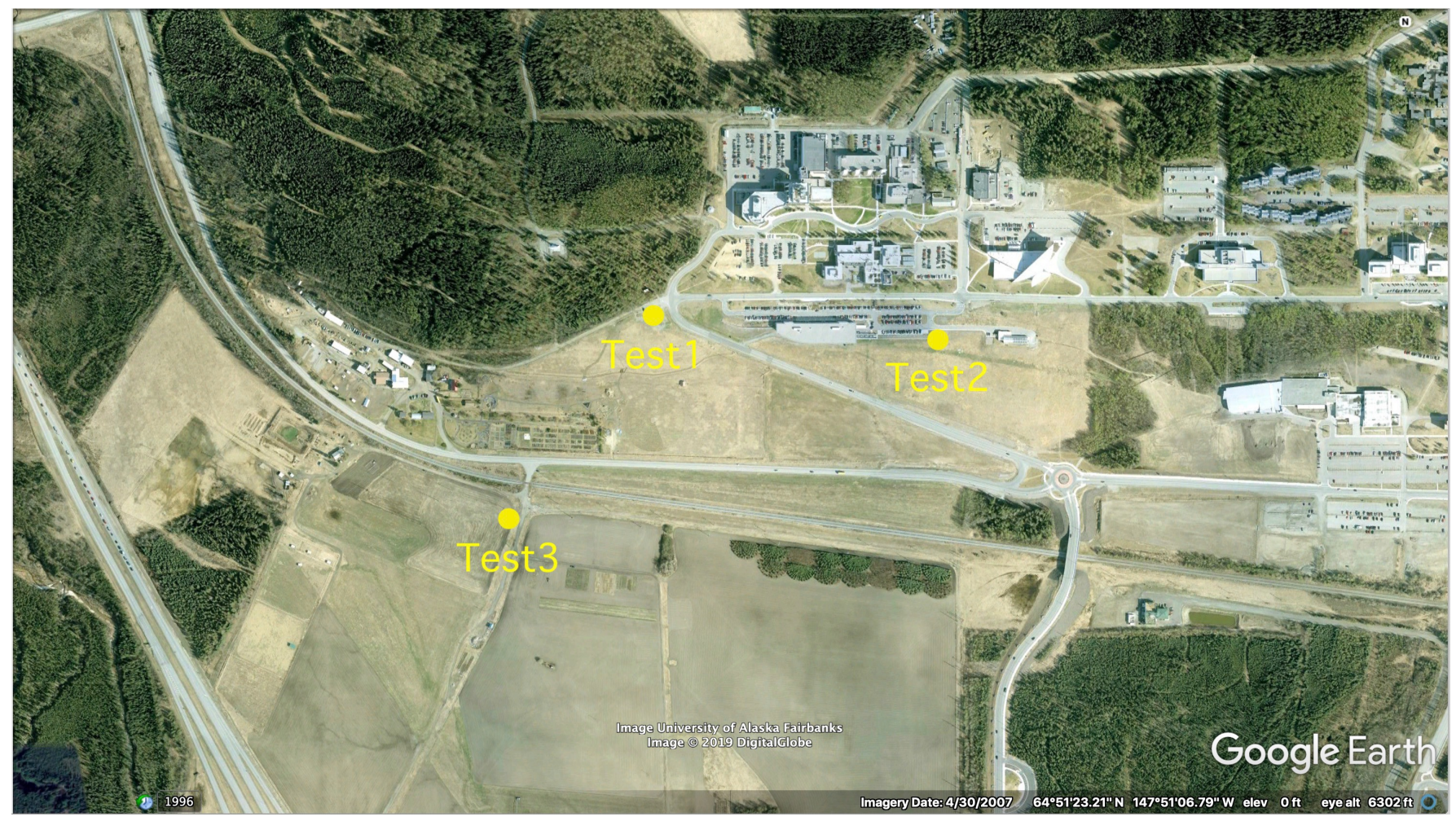

Figure 1. Alaska Test Location

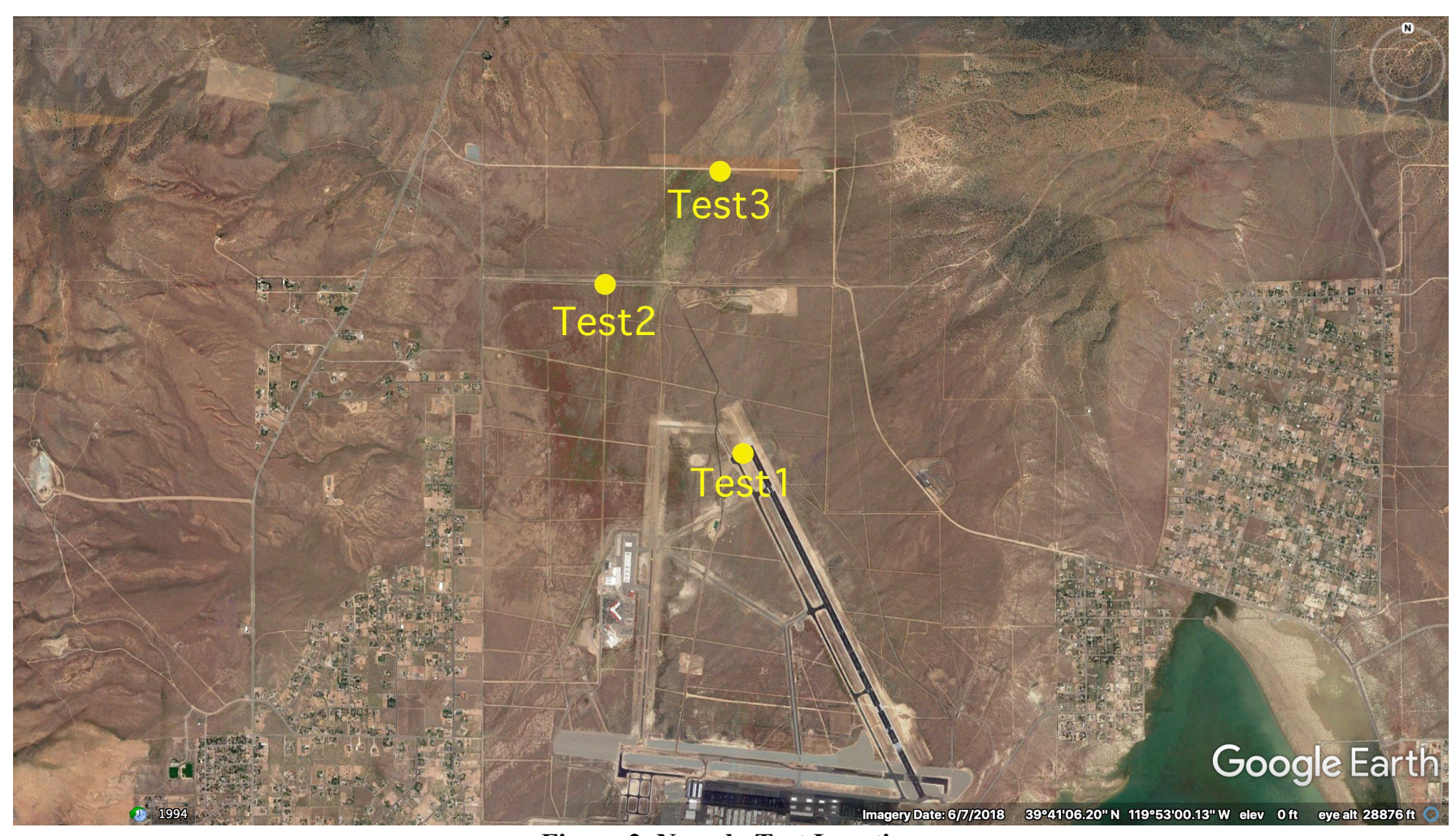

Figure 2. Nevada Test Location 


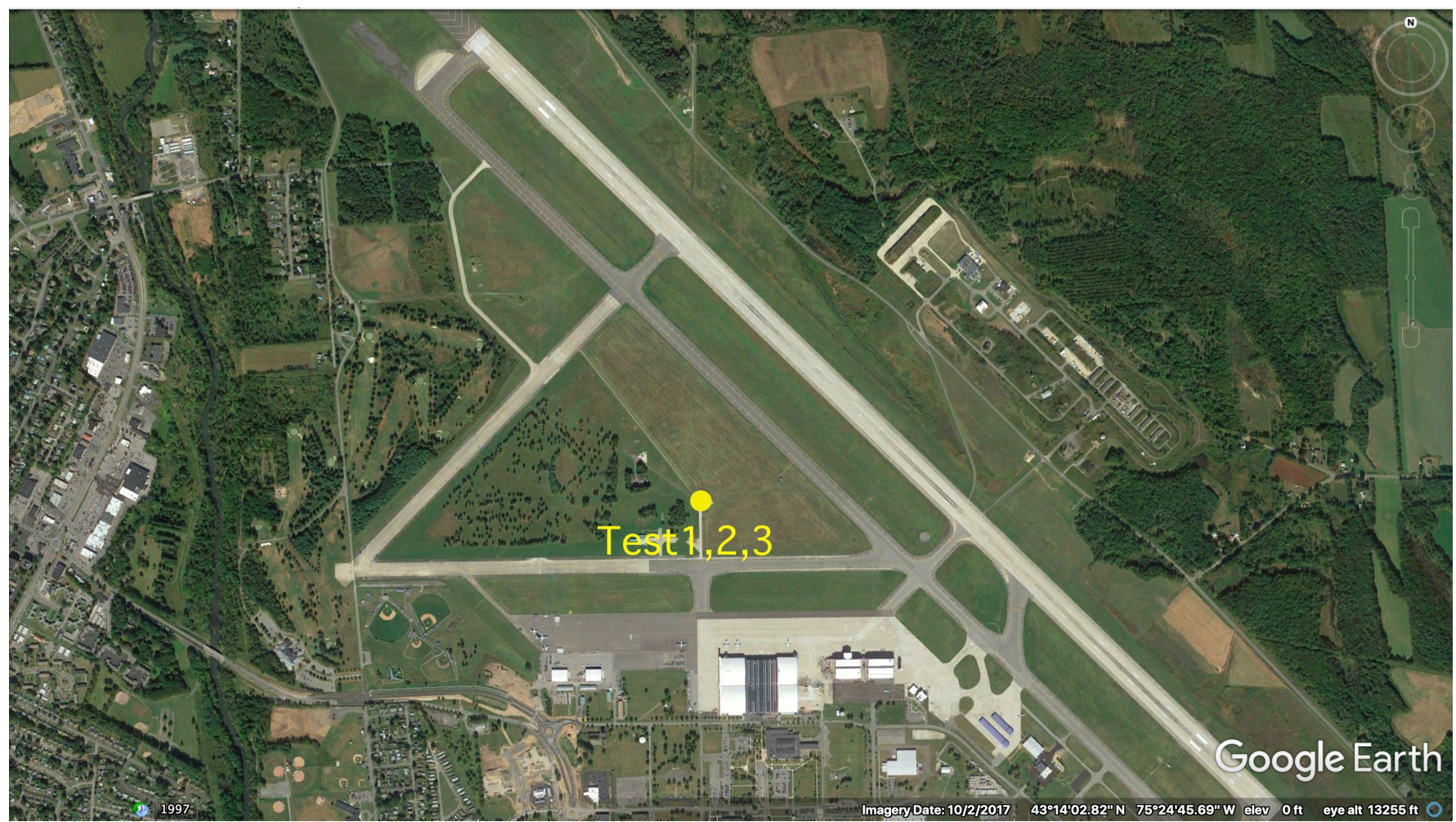

Figure 3. New York Test Location

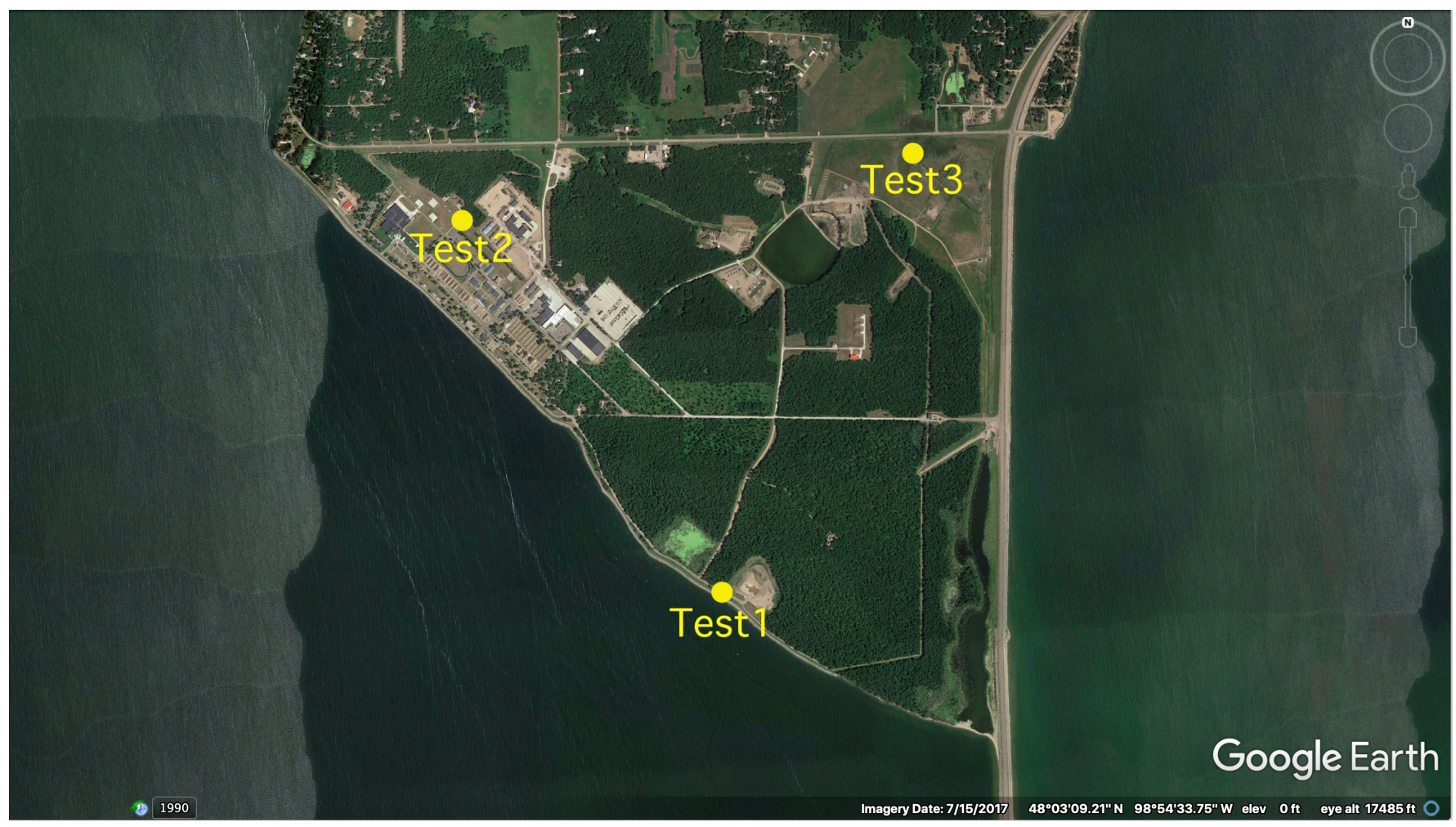

Figure 4. North Dakota Test Location 
LTE cellular modem was flown in Rome. Figure 3 shows the flight location [9]. The flight was designed to bring the UA outside of $\mathrm{Wi}-\mathrm{Fi}$ range and when this occurred, C2 communications were automatically transferred to the LTE modem. The New York TSO reported that no issues occurred during this transfer and the operator maintained control.

\section{North Dakota}

The North Dakota flight test was conducted on April 11-12, 2018. A helicopter UA equipped with two different radio modems, a fixed-wing equipped with a radio modem and a satellite communications modem, and a hexacopter equipped with a radio modem and an LTE cellular modem were flown in Camp Grafton. Figure 4 shows flight locations [10]. The three UA were flown in two conditions. In the first condition, the operator manually disconnected one $\mathrm{C} 2$ communications link to force switching. In the second condition, the UA has flown a long distance where one $\mathrm{C} 2$ link failure occurred without intervention from the operator. The North Dakota TSO reported that although there was a latency of about 50 seconds in switching to the satellite communications modem, this was known beforehand and did not impact the operator's control of UA in both test conditions.

\section{RECOMMENDATIONS FOR CONFIGURING REDUNDANT C2 COMMUNICATIONS SYSTEM FOR URBAN OPERATIONS}

The next phase of the UTM research focuses on sUAS operations in an urban area. Compared to a rural area, one can expect an increased level of RF activities across a broad spectrum to take place in an urban area due to wireless voice and data communications, broadcasting venues, and electronics used by a larger, concentrated population [11]. Also, highdensity, fast-tempo sUAS operations carrying out tasks such as newsgathering and package deliveries are expected to take place in the airspace over an urban area in the future. Therefore, the following are recommended to assist sUAS operators for configuring an effective redundant $\mathrm{C} 2$ communications system to maintain operational control during urban operations.

Recommendation 1: The operator should not use C2 systems that use the ISM radio bands for redundancy as they are not reliable in an urban setting.

Although the tested redundant $\mathrm{C} 2$ communications configurations, shown in Table 2, were found effective in maintaining operational control of sUAS in the airspace over rural locations, all configurations include C2 systems that use the industrial, scientific and medical (ISM) radio bands, such as $902 \mathrm{MHz} \sim 928 \mathrm{MHz}$ and $2.4 \mathrm{GHz} \sim 2.5 \mathrm{GHz}$ [12]. The ISM bands can be used freely within the regulated transmit power limit [13] and due to its open nature, C2 communications systems using the ISM bands can experience interference from applications, such as Wi-Fi routers, at any time in an urban area. As another example, radio energy from everyday electronics, such as a microwave oven, can be released to the ISM radio bands to interfere with the $\mathrm{C} 2$ systems using the same bands, and this phenomenon is likely more intense in an urban area than in a rural area. Therefore, for urban operations, C2 systems that use ISM radio bands should not be included in redundancy configurations as they will be subjected to difficult-to-predict interferences and will not be reliable.

Recommendation 2: The operator should verify the RF characteristics of the intended operation area and examine the radio noise floor during operations.

To configure a redundant $\mathrm{C} 2$ communications system that is robust to RF interferences, the operator should obtain the RF characteristics in the intended operation area, such as locations of broadcasting antennae, transmit frequency bands, power, and schedule. Thereafter, the operator should configure redundancy by including $\mathrm{C} 2$ systems using frequencies that are away from the known high-powered transmission frequencies, unless the high-powered transmission is for the $\mathrm{C} 2$ communications. Once the redundant $\mathrm{C} 2$ communications system is configured, it is prudent for the operator to check the noise floor of all $\mathrm{C} 2$ communications frequencies in the operation area. When noise floor increases in one radio band, the operator can switch $\mathrm{C} 2$ communications to the other band to proactively prevent loss of communications and maintain control. Also, it is not recommended to feature two identical systems in the redundancy, such as two identical radio modems using the same frequency bands. While this arrangement can be effective in mitigating a communications system malfunction, RF interference affecting one system will affect the other, negating redundancy.

Recommendation 3. The operator should monitor the availability, quality, and reliability of communications services that are used by the redundant system.

When the redundant part of the $\mathrm{C} 2$ system uses an external service, such as LTE cellular and satellite communications, redundancy is maintained only when the service is reliably available in the required quality. Therefore, it will be necessary for the operator to know whether the required service is available in the intended operation area and have a mechanism to keep track of the service quality and reliability. For this monitoring to be effective, the operator must know the performance of the communications required for the intended operation, such as minimum data transfer rate and maximum tolerable latency. When the monitoring finds the performance of one system below-required, switching to the other system should happen within the maximum tolerable latency so that operational control is maintained during this switching. Therefore, the operator should not include a C2 system that requires switching time longer than the maximum latency. For example, if switching to satellite communications requires 50 seconds, as reported by the North Dakota TSO, the operator must exclude such a system from the redundancy configuration if the 50 seconds is larger than the maximum tolerable latency.

Recommendation 4. The sUAS community should adopt a standard set of contingency steps to manage the loss of $\mathrm{C2}$ communications in a consistent manner across the airspace.

Loss of $\mathrm{C} 2$ communications is identified when all $\mathrm{C} 2$ communications systems in a redundancy configuration 


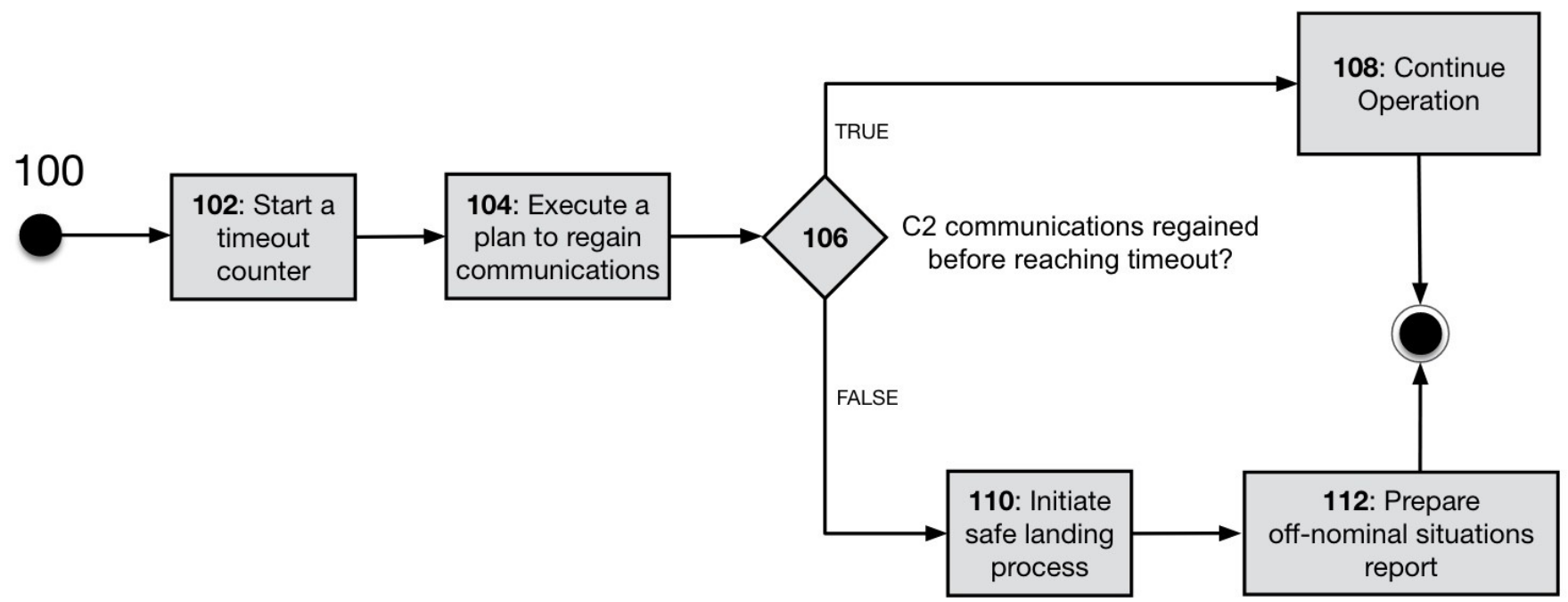

Figure 5. High-Level Loss of C2 Communications Mitigation Steps

experience persistent below-required performance. This can occur due to broad-spectrum RF interference, hardware malfunction, etc. As high-density sUAS operations are envisioned to take place in the airspace over an urban area, when Loss of $\mathrm{C} 2$ communications occurs in one sUAS operation it will be important for that sUAS to display predictable behavior. This will facilitate other sUAS in the vicinity to anticipate an impact on their operation and take necessary measures to minimize any interruptions.

High-level mitigation steps to manage a Loss of $\mathrm{C} 2$ communications situation in a consistent manner are presented in Fig. 5 for adoption and further development by the sUAS community. To instill a predictable behavior while giving the freedom to operators to design a method to regain communications as they see fit, a notion of "timeout" is introduced in these steps. That is, sUAS should be programmed to regain communications when Loss of $\mathrm{C} 2$ communications occur until the timeout is reached, and upon the timeout initiate safe landing process.

100: Loss of $\mathrm{C} 2$ Communications condition identified.

102: Start counting time in Loss of $\mathrm{C} 2$ Communications condition, to compare against set timeout value at $\mathbf{1 0 6}$.

104: Execute a plan to regain $C 2$ communications. Example plan: fly higher and loiter for better reception, etc.

106: Compare the elapsed time in Loss of $\mathrm{C} 2$ Communications condition to the timeout value.

108: Continue nominal operation.

110: UA initiates a safe landing process. A safe landing is achieved when UA lands without causing a detrimental impact on people and property.

112: The operator prepares a report that contains offnominal situation details.

The length of the timeout is to be specified per a type of operation and its environment. For example, the length of timeout for solo operation over a cornfield can be much longer than one for high-density operation over a city as Loss of C2 Communications poses less risk for the former than the latter. Discussion about the safe landing process is out of the scope of this paper and will be addressed in other UTM documentation.

\section{CONCLUSION}

Flight tests showed that the redundant $\mathrm{C} 2$ communications systems implemented by four UAS test sites were effective in maintaining operational control of sUAS in the airspace over several rural locations, as defined by the operators. Based on the test experience, four recommendations are made to assist sUAS operators for configuring an effective redundant $\mathrm{C} 2$ communications system to maintain control in the airspace over an urban area where an increased level of RF activities takes place compared to a rural area. The NASA FAA UTM research team will use insights from the test to accommodate the FAA's UAS integration effort.

\section{ACKNOWLEDGMENT}

The authors thank test site operators and their partners for conducting safe and successful flight tests. Also, appreciations go to the NASA UTM team.

\section{REFERENCES}

[1] Kopardekar, P., "Unmanned Aerial System Traffic Management (UTM): Enabling Low-altitude Airspace and UAS Operations," Tech. Rep. NASA TM-2014-21829, 2014.

[2] Kopardekar, P., Rios, J., Prevot, T., Johnson, M., Jung, J., and Robinson, J. E., "Unmanned Aircraft System Traffic Management (UTM) Concept of Operations," 16th AIAA Aviation Technology, Integration, and Operations Conference, Washington, D.C., 13-17 June 2016.

[3] FAA UAS Traffic Management [Online], Accessed: June 7, 2019. https://www.faa.gov/uas/research_development/traffic_management/

[4] Unmanned Aircraft System (UAS) Traffic Management (UTM) Concept of Operations v1.0, the FAA, May 2018.

[5] UAS Traffic Management (UTM) Research Transition Team (RTT) Plan, NASA and the FAA, January 2017. 
[6] Leck, R., "Results of Ambient RF Environment and Noise Floor Measurements Taken in the U.S. in 2004 and 2005", World Meteorological Organization, CBS/SG-RFC 2005/Doc. 5(1), March 2006.

[7] Google Earth, Fairbanks, Alaska Area Sattlite View, Accessed: July 11, 2019.

[8] Google Earth, North Valleys, Neveda Area Satellite View, Accessed: July 11, 2019.

[9] Google Earth, Rome, New York Area Satellite View, Accessed: July 11, 2019.
[10] Google Earth, Camp Grafton, North Dakota area view, AccessedL July $11,2019$.

[11] Enge, P., Akos, D., and Do, J., "Measurement of Man-Made Spectrum Noise Floor", NASA CR-2004-213551, November 2004.

[12] National Telecommunications and Information Administration Tables of Frequency Allocations (27.5 - $10000 \mathrm{MHz}$ ) [Online], Accessed: June 28, 2019. https://www.ntia.doc.gov/legacy/osmhome/alloctbl/allocmhz.html

[13] Federal Communications Commission, Code of Federal Regulations Title 47 Part 15. 